\title{
The Testing Methods Research on Athletes Nutrition and Supplement of Anaerobic Endurance Track and Field Events Based on Wireless Scanning Technology
}

\author{
Caigui Wan \\ College of Physical Education, Jiangxi Science and Technology Normal University, Nanchang \\ Jiangxi, 330013, China
}

Key Words: Multiple Source, Wireless Scanning Data, Athletes Nutrition and supplement Testing of Anaerobic Endurance Track and Field Events, The reliability of Nutrient Sources.

\begin{abstract}
It is widely researched and confirmed in traditional track and field events that the athletes nutrition and supplement testing of anaerobic endurance track and field events could make nutritional integration which may come from different nutrient sources. However, some existing athletes nutrition and supplement testing methods could not adapt the application of Wireless Scanning Technology Flow for the reason that these existing methods include iterative process. As far as the Wireless Scanning Technology Flow is concerned, this technology flow has obvious characteristics and also make an survey on athletes nutrition and supplement testing of anaerobic endurance track and field events from the perspective of data flow. On this basis, this article could make effective use of wireless scanning data and also advance strategies which could make a frequency conversion evaluation on reliability of nutrient sources, and this strategy could decrease these required iterative process, and also increase efficiency during different time. And this article proposes a algorithm named HQW which could be used to make frequency conversion evaluation on reliability of nutrient sources. The HQW combines historical data and determines the evaluation moment of reliability of nutrient sources dynamically, this algorithm not only insure the athletes nutrition and supplement testing of anaerobic endurance track and field events could research the standard, but also increase efficiency. At last, this paper further validates the efficiency and accuracy of HQW on perception and handling of athletes nutrition and supplement testing by wireless scanning technology flow through the experiment on the real wireless scanning data set.
\end{abstract}

\section{Introduction}

If there exist some different explanations on identical entity which under the influence of different nutrient, then the real description about this entity requires effective integration of these information, and this procedure is called the process of athletes nutrition and supplement testing of anaerobic endurance track and field events (Truth Discovery). In recent years, the athletes nutrition and supplement testing of anaerobic endurance track and field events have been in-depth researched in the field of traditional track and field events ${ }^{[1-3]}$. However, with the development of network information technology, computer science, mobile technology, a large amount of application models of wireless scanning technology flow appear in many fields, which including communication management, financial application, data processing of sensor and so on. Which worth the whistle is that the data is in the state of variation during the application of wireless scanning technology. And also the nutritive value should be changed accordingly, which makes it necessary to test athletes nutrition and supplement of anaerobic endurance track and field events on these new data of every moment during the research process of nutritional values. 
The major study context is the athletes nutrition and supplement testing of anaerobic endurance track and field events which based on wireless scanning technology, especially the development of wireless communication technology and micro-electronics, and also the continuously increase of the study about wireless sensing technology. The sensor network consists of numerous sensor nodes which located in particular block, and every node has function of information calculation, information storage and information communication. That is to say that these athletes could inquire these information in coverage area by wireless sensor network. However, this problem that athletes nutrition and supplement testing of anaerobic endurance track and field events has not received adequate critical emphasis.

\section{The Testing Methods of Athletes Nutrition and Supplement of Anaerobic Endurance Track and Field Events which Based on Wireless Scanning Technology}

In the fact, the nutrient source weight of next moment could not be obtained at current moment because of uncertainty. Therefore, it is difficult to determine the fluctuation of weight value. This article proposes the possibility prediction model on the basis of Bernoulli distribution, and also could determine if the predicted fluctuation of nutrient source weight value match the possibility in Formula(1) by this model.

At first, set a certain threshold value ' $\varepsilon$ ' . For the reason that the credibility evaluation of nutrient source and the arrival time of data are independent of each other, or in other words that these events are independent and random when make an judgment about the matching degree of weight fluctuation and Formula (1), and there are also two results that either establishment or not. As a consequence, this judgment process could be regarded as Bernoulli process, that is to say every judgment event should comply with Bernoulli distribution $\xi \sim B(1, p)$. And the experiments section is verified. And the following part is started from the efficiency estimation of $p$.

Case One : Suppose that there are $\mathrm{K}$ nutrient sources. If the weight renovation of nutrient source is achieved by $\mathrm{CRH}$ algorithm in anytime of $t_{i 0} \sim t_{j 0}$, then the weight fluctuation in anytime could be in line with the graph (1), which is presented by $N_{0}$. The averaging time in this time horizon is $M_{0}\left(M_{0}=j_{0}-i_{0}\right)$, and the probability is that $\hat{p}=N_{0} / M_{0}$. However, the weight value fluctuation could be influenced by energy consumption and external affecting as time goes on, and the weight value fluctuation of nutrient sources changes over. Thus the result accuracy could be increased drastically if the dynamical estimation be achieved.

According to the above mentioned probability model, if predict that all the weight fluctuation of nutrient source could conform to $\beta$ in Formula (1) over a period of time ${ }^{t_{i+1}}, t^{t_{+2}}, \ldots,{ }^{t_{j}}$, then the probability of cumulative error in this period of time must be equal or greater than $\beta$, among $\Delta T=j-i$. As a result, if the prior regeneration point could be grasped accurately, and the weight fluctuation of nutrient source in a period of time could achieve the probability requirement of Formula (1), and make a prediction on the maximum of cumulative error, then could predict the next regeneration point accurately. Meanwhile, the time interval between each regeneration point are likely to be different from the perspective of dynamic renewal nature of nutrient source.

To illustrate the major stage of AVF algorithm by the combination with Graph 1.

Update the weight of nutrient source: through CRH algorithm, update the weight of nutrient source $W_{i}, W_{i+1}$ of the known regeneration point $t_{i}, t_{i+1}$;

Update the probability $\hat{p}$ : make an statistics $\Delta w_{i+1}^{k}(1 \leqslant k \leqslant K)$ by $W_{i}, W_{i+1}$, and get the renewal probability $\hat{p}$;

Predict the next regeneration point: get ${ }^{t_{j}}$ mainly by solving of optimization problem of Formula (4). 


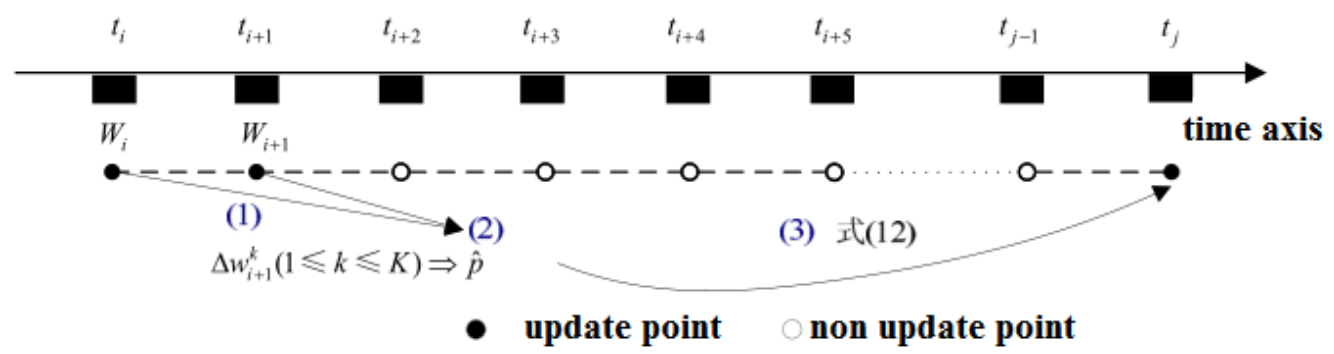

Fig. 1. The process of HQW

The parameters of HQW algorithm consists of $\alpha, \varepsilon$ and $\gamma$, so the parameter setting is important:

The threshold value of probability $\alpha . \alpha$ is mainly restriction, it means the minimum probability which satisfies all the weight fluctuation of nutrient source in a given time period. The smaller the $\alpha$ is, the larger the $\Delta T$ is, and the fewer the required number of evaluation on weight value of nutrient source is, and the higher the efficiency is; On the contrary, the lower the efficiency is.

The threshold value of accumulative error ${ }^{\gamma}{ }^{\gamma}$ restricts the maximum of accumulative error mainly. The maximum refers to the predicted maximum in a given period, this is also called the upper limit. If the smaller the ${ }^{\gamma}$ is, then the higher the required accuracy of determination of athletes nutrient and supplement, the larger the required number of evaluation on weight value of nutrient source is, it refers to that the efficiency of algorithm under this circumstance is low, but the accuracy is relatively high; otherwise, the accuracy is relatively low, but the efficiency is relatively high.

The threshold value of relative error $\varepsilon$. $\varepsilon$ restricts the relative error between adjoining times. The larger the $\varepsilon$ is, the higher the weight fluctuation between adjoining times is, otherwise it proves that the adjoining time has relative stable weight fluctuation. According to Formula (4), the influence of $\varepsilon$ fluctuation on the accuracy and efficiency of AVF algorithm is not unchangeable. If the weight fluctuation is high, then the higher the $\varepsilon$ is, the higher the accuracy of AVF algorithm is, on the contrary, if the weight fluctuation is low, then the higher the $\varepsilon$ is, the fewer the efficiency of AVF algorithm is, the higher the accuracy is.

In conclusion, the performance of AVF algorithm could be changed by adjusting the parameter setting, and the relevant requirements of efficiency and accuracy could be realized. And this article make a verification on this influence by the subsequent experiment section.

\section{The Analysis of Experiment and Result}

The aggregate of the wireless scanning data which used in this experiment is authentic, and the performance of AVF algorithm is analyzed and verified on this basis. In combination with experimental result, the hypothesis of probability distribution is reasonable and logical. In the respect of testing problems about athletes nutrition and supplement in anaerobic endurance track and field events which based on wireless scanning technology flow, as the AVF algorithm demonstrated, the accuracy and efficiency are relatively good, and AVF algorithm could perceive the arrival speed of wireless scanning technology flow and has faster processing speed.

\section{Experimental Result}

As the graph 2 shows, it reflects the accuracy fluctuation of HQW algorithm when the parameter value is $\alpha, \varepsilon, \gamma$ respectively. And meanwhile compare these values with GTM algorithm. Set each parameter as follow:

(a) $\varepsilon=5 \times 10^{-4}, \gamma=1, \alpha$ are $0.65,0.75,0.85$ respectively;

(b) $\alpha=0.7, \gamma=0.1, \varepsilon$ are $0.005,0.05$ respectively; 


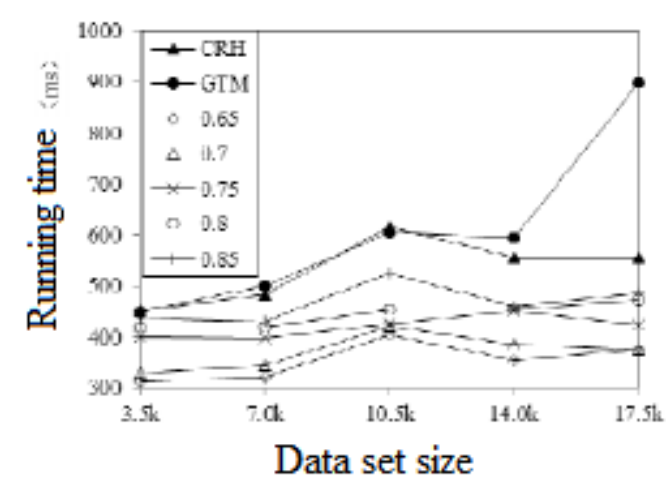

(a) $\alpha$ changes

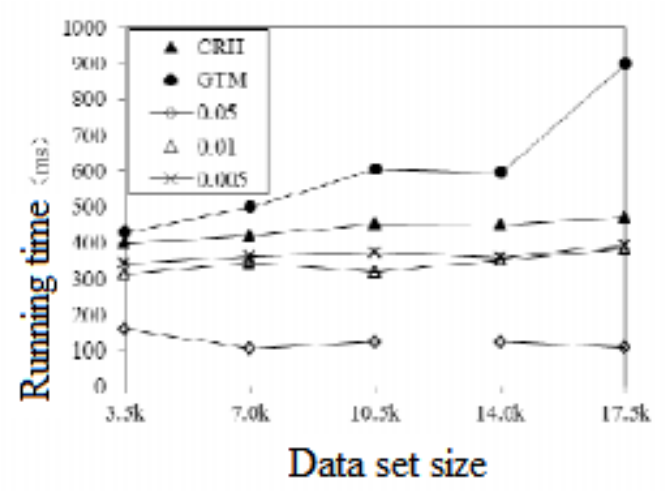

(b) $\varepsilon$ changes

Fig. 2. The wireless scanning technology

Analyze the wireless scanning technology, if the number of renewals caused by corresponding parameter is frequent, then the accuracy is higher. By analyzing the Graph 2, this data set has a high weight fluctuation, and when $\alpha=0.65$, its renewal number is large, that is to say that if data set has a great weight fluctuation, then the influence of $\alpha$ on renewal number is relative finite. Thus, if the parameter range of $\alpha$ in Graph 2 changes, the influence on accuracy of HQW algorithm is not obvious. And AVF algorithm adopts CRH algorithm in the regeneration point. Comparing with GTM algorithm, CRH algorithm has a higher static data set. Therefore, comparing with GEM algorithm, even though the HQW algorithm need to update weight value of nutrient source continuously, but the regeneration point of HQW algorithm has a great accuracy, so the accuracy of HQW algorithm is guaranteed. Meanwhile,the GEM algorithm in Intel Berkeley laboratory data set that the mean square error could be increased with the escalation of data set, but the accuracy of HQW algorithm could be decreased gradually. This reveals that the HQW algorithm has an advantage over the perception of wireless scanning technology flow.

The performance of algorithm has been verified by these above experiments. And through experimental analysis, these two sets of data set has obvious dissimilarity, and it further proves that the HQW algorithm has great performance on handling with data sets which have different models and types.

\section{The result}

In traditional data, the application researches on athletes nutrition and supplement testing of anaerobic endurance track and field events are fairly common, especially in the aspect of dispelling these data inheritance conflict. However, based on the restrictions of time and space, this type of technology which are applied in traditional data field is faced up with hardships when it extends to wireless scanning technology flow. This paper starts from the perception of wireless scanning technology flow which is the specific form of wireless scanning technology flow, and make an study on athletes nutrition and supplement testing of anaerobic endurance track and field events. Integrate the application characteristics of wireless scanning data, and propose that make a frequency conversion estimation, and make an balance with accuracy and efficiency. At first, define the relative error and accumulative error, and explain these required criteria of fluctuation condition of feasible degree during imminent period, and propose probability model, and then predict the probability. At last of this paper, integrate these correlation theories and achieve effective adoption of the athletes nutrition and supplement testing problem of anaerobic endurance track and field events in wireless scanning technology, and obtain a optimization problem. And then through the proper restriction on accumulative error, maximize the assessment period of nutrient source reliability and meanwhile improve the efficiency. 


\section{References}

[1] Yin XX, Han JW, Yu PS. Truth discovery with multiple conflicting information providers on the Web. IEEE Trans. on Knowledge and Data Engineering, 2016,20(6):796-808.

[2] Galland A, Abiteboul S, Marian A, Senellart P. Corroborating information from disagreeing views. In: Proc. of the WSDM. New York, 2014. 131-140.

[3] Zhao B, Han JW. A probabilistic model for estimating real-valued truth from conflicting sources. In: Proc. of the QDB. Istanbul, 2012. 